\title{
Numerical simulation on the marine landslide due to gas hydrate dissociation
}

\author{
X. B. Lu ${ }^{1,2} \cdot$ X. D. Chen ${ }^{1}$ L. Lu ${ }^{1,3} \cdot$ X. H. Zhang ${ }^{1}$
}

Received: 14 March 2016/Accepted: 7 February 2017/Published online: 18 February 2017

(C) Springer-Verlag Berlin Heidelberg 2017

\begin{abstract}
Marine landslide due to gas hydrate dissociation is a kind of potential heavy hazards. Numerical simulation was processed to investigate the deformation, landslide and effects of main factors on the critical scale of dissociation zone to induce marine landslides during/after dissociation of gas hydrate. A simple method for analyzing the critical scale was presented based on the limit equilibrium method. It is shown that the maximum settlement is located near the upper side of the dissociation zone. The soil near the lower side of the dissociation zone uplifts. There is a critical scale of dissociation zone over which landslide will occur under given conditions. Expansion modes of the dissociation zone have obvious effects on the critical scale.
\end{abstract}

Keywords Gas hydrate - Dissociation zone - Marine landslide

\section{Introduction}

Natural gas hydrate (NGH) is a kind of crystalline solid formed by methane gas and water under high pressure and low temperature. It is presented in ocean sediments, continental margins and deep lakes, containing more than half

X. B. $\mathrm{Lu}$

xblu@imech.ac.cn

1 Institute of Mechanics, Chinese Academy of Sciences, Beijing 100190, China

2 Department of Engineering Sciences, University of Chinese Academy of Sciences, Beijing 100049, China

3 School of Science, China University of Mining and Technology, Beijing 100083, China of the organic carbon in the Earth. Thus, NGH is taken as a future strategic energy resource (Sloan 1998).

NGH can dissociate with either the rise of temperature or the decrease in pressure, such as the drop of sea level, petroleum drilling/production operations (Briaud and Chaouch 1997), which will directly reduce the strength of hydrate-bearing sediment (HBS). Generally, about $164 \mathrm{~m}^{3}$ methane gas at standard conditions and $0.8 \mathrm{~m}^{3}$ water can be released from only $1 \mathrm{~m}^{3} \mathrm{NGH}$. The excess pore pressure will increase and the sediment's strength will decrease if the released gas cannot drain quickly, which can cause kinds of disasters, such as destruction of ocean platforms, oil wells or even gas blowouts (Milkov 2000; Xu and Germanovich 2006; Zhang et al. 2010, 2011; Chaouch and Briaud 1997). For example, some studies showed that the Storegga landslide on the Norwegian continental shelf, the largest landslide in the world with $2500-3200 \mathrm{~m}^{3}$ sediments brought away, was induced by NGH dissociation (Bugge et al. 1987). NGH dissociation is also thought to be the reason of the US mid-Atlantic coast slide (Jung and Peter 2004).

Hydrocarbon exploration and production are now extending gradually to the area existing NGH. There is a concern that these activities can trigger NGH dissociation and consequent marine landslides or offshore structures' damage (Briaud and Chaouch 1997; Zhang et al. 2010; Kwon et al. 2008; Brooks et al. 1986). For example, formation and dissociation of NGH were thought to be the main reason of the Deepwater Horizon explosion happened years ago in the Gulf Mexico because the sediments that the drilling rig had reached were just suitable for $\mathrm{NGH}$ formation (Marcia et al. 2012).

Though the methane gas extraction from HBS has been studied by coupling NGH dissociation, seepage and heat conduction ( $\mathrm{Lu}$ et al. 2010; Wang et al. 2009; $\mathrm{Li}$ and $\mathrm{He}$ 
2012), the initiation and the patterns of the sediment failure due to NGH dissociation are still required to study deeply.

In this report, commercial software FLAC3D is adopted to study the sediment's responses due to NGH dissociation. The aim is to study the deformation and slide of the ocean bed after NGH dissociation. Effects of main factors (elastic modulus, internal friction angle, cohesion, water depth, height ratio of NGH layer and over layer and the expansion mode of dissociation zone) are discussed. A simple method for analyzing the critical scale of dissociation zone to cause landslide is given based on limit equilibrium method.

\section{Numerical model}

The concerned problem is: The heat for NGH dissociation is provided by the hot fluids flowing through a vertical wellbore/pipe in the center of the considered zone. NGH dissociation starts from the wellbore/pipe and expands cylindrically gradually. The strength and modulus of the sediment decrease and deform happens with NGH dissociation. When the dissociation zone expands to be greater than some critical diameter (CD), the slope will slide because the decreased strength cannot resist the sliding force. The aim of the numerical simulation is to investigate the effects of the main factors on the deformation, initiation and critical conditions of landslide.

Software fast lagrangian analysis of continua (FLAC3D) is developed by the Itasac Consulting Group Inc. and University of Minnesota in USA which is appropriate for the analysis of geotechnical and geological engineering. This software can conveniently simulate the plastic failure and large-scale deformation and distortion by using the mixed discretization technique, dynamic relaxation method, finite difference method, especially the Lagrange algorithm. The adoption of explicit algorithm leads the progressive failure of sediment to be traced easily, which is very important for engineering.

The sediment considered for numerical simulation was three-dimensional and consisted of three layers: The upper (over layer) was impermeable soft soil with thickness $(h)$ of $200 \mathrm{~m}$ and the middle was HBS with thickness $(H)$ of $100 \mathrm{~m}$ and the lower was hard rock with thickness $100 \mathrm{~m}$. The length along slope was $5000 \mathrm{~m}$, and the width was $1000 \mathrm{~m}$.

The vertical wellbore/pipe located at the center was modeled by pile elements provided by FLAC3D. The sediment was modeled by using meshes with size of length $\times$ width $\times$ height $=10 \mathrm{~m} \times 10 \mathrm{~m} \times 5 \mathrm{~m}$, respectively. Contact elements were adopted for modeling the interface between the wellbore/pipe and sediment. The strength parameters (internal friction angle and cohesion) of the contact element were chosen to equal that of the sediment. Thus, the sediment and wellbore/pipe were permeated to separate during deformation. The surface of the sediment was assumed to be free that means the effective stresses at the surface were all zero. The other boundaries were all normally fixed. So layers at the upper and lower ends were permitted to settle. The displacements and stresses at the interface between the over layer and HBS were continuous before failure. The sketch for numerical simulation is shown in Fig. 1.

The overall instability of the slope was judged by either of the two conditions: (1) The plastic zone was connected along slope and formed a slide face. (2) Deformation of any point in the slope could not stop.

\section{Controlling equations}

The controlling equation for the deformation of sediment was adopted as.

$\rho \frac{\mathrm{d} u_{i}}{\mathrm{~d} t}=\frac{\partial \sigma_{i j}}{\partial x_{j}}+\rho g_{i}$

in which $\rho=(1-n) \rho_{\mathrm{s}}+n \rho_{\mathrm{w}}$ is the density of sediment, $\rho_{\mathrm{w}}$ and $\rho_{\mathrm{s}}$ are densities of fluid in pores and skeleton, $i$, $j$ denote the tensor indexes, $g$ is the gravity acceleration.

The incremental form of the constitutive relationship provided by FLAC3D was adopted:

$\left\{\begin{array}{l}\Delta \sigma_{1}=\alpha_{1} \Delta \varepsilon_{1}+\alpha_{2}\left(\Delta \varepsilon_{2}+\Delta \varepsilon_{3}\right) \\ \Delta \sigma_{2}=\alpha_{1} \Delta \varepsilon_{2}+\alpha_{2}\left(\Delta \varepsilon_{1}+\Delta \varepsilon_{3}\right) \\ \Delta \sigma_{3}=\alpha_{1} \Delta \varepsilon_{3}+\alpha_{2}\left(\Delta \varepsilon_{1}+\Delta \varepsilon_{2}\right)\end{array}\right.$

in which $\Delta \sigma_{i}(i=1,2,3)$ is the increment of current effective stress, $\Delta \varepsilon_{i}(i=1,2,3)$ is the increment of strain, $\alpha_{1}=\mathrm{K}+4 \mathrm{G} / 3$ and $\alpha_{2}=\mathrm{K}-2 \mathrm{G} / 3, \mathrm{~K}$ is the volume modulus and $G$ shear modulus.

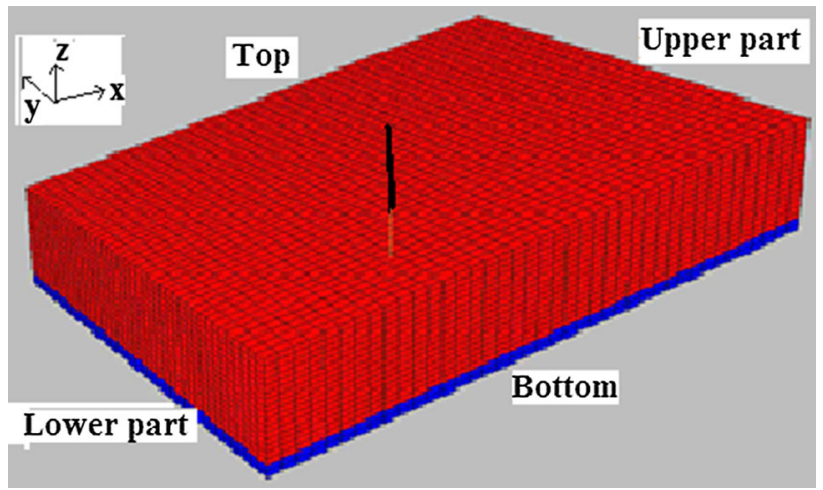

Fig. 1 Computational domain (the wellbore is located at the center of the computing zone vertically (black line). The $X$-axis is in horizontal direction, and $Y$-axis is in vertical direction and parallel to the wellbore/pipe) 
HBS can be thought as a kind of special soil in which $\mathrm{NGH}$ is a part of the skeleton which can bear load and meanwhile a kind of binder to cement soil grains. Thus, HBS must have internal friction and cohesion. The MohrColumn constitutive relation is shown to be suitable for describing the HBS behavior (Zhang et al. 2012) and was used in the numerical simulation:

$\tau_{\mathrm{f}}=\sigma \tan \varphi+C$

in which $\tau_{\mathrm{f}}$ is the shear strength, $\sigma$ is the effective stress on the considered face, $\phi$ is the internal frictional angle, $C$ is the cohesion.

Because the propagation of stress and seepage in the sandy sediment is very faster than the heat conduction and NGH dissociation (Zhang et al. 2010), the changes of the stress and displacement and the seepage were assumed to happen immediately after NGH dissociation. The dissociation was finished immediately after the heat supply, and so the change front of the seepage was the same as that of the heat conduction. There was no partial dissociation zone in the sediment. The dissociation zone started from the vertical center line of the sediment and expanded away with a velocity determined by the heat conduction and seepage (Zhang et al. 2010; Liu et al. 2015) since NGH dissociation was caused by the heat conducted from a vertical pipe or wellbore. In computation, the dissociation zone was assumed to expand with a length $(=10 \mathrm{~m})$ equaling to the distance between two grids along slope once the heat front had reached. Such processing is convenient for numerical simulation and the induced error can be neglected because this length is enough small relative to the whole length of the considered area $5000 \mathrm{~m}$.

The mechanical properties of HBS were obtained by triaxial shear tests (Zheng et al. 2004; Lu et al. 2008). For the over layer, they were as follows: Elastic modulus $E=1 \mathrm{MPa}$, cohesion $C=10 \mathrm{kN}$, internal friction angle $\varphi=20^{\circ}$, density $\rho=2000 \mathrm{~kg} / \mathrm{m}^{3}$; for HBS before NGH dissociation: $E=186 \mathrm{MPa}, \quad C=0 \mathrm{kN}, \quad \varphi=39.4^{\circ}$, $\rho=2150 \mathrm{~kg} / \mathrm{m}^{3}$. The material parameters of the sediment were thought to change directly from the value before NGH dissociation to that after NGH dissociation, because the stress and seepage were assumed to be finished very shortly after the heat supply and NGH dissociation. To investigate the effects of the relative changes of main factors (height ratio of sediment, cohesion and internal friction angle of HBS after NGH dissociation, height ratio of water) on the responses of the slope, four different values were chosen for each material factor while the other parameters kept as constants. Internal friction angle and cohesion are the strength parameters of HBS after NGH dissociation, height ratio of sediment indicates the effects of gravity, and the height ratio of water indicates the effects of external load. For example, four values 2.0, 1.0, 0.67 and
Table 1 Parameters adopted in numerical simulation

\begin{tabular}{lllll}
\hline No. & $\begin{array}{l}\text { Height ratio of } \\
\text { sediment }(H / h)\end{array}$ & $\begin{array}{l}\text { Internal } \\
\text { friction angle } \\
\left({ }^{\circ}\right)\end{array}$ & $\begin{array}{l}\text { Cohesion } \\
(\mathrm{Pa})\end{array}$ & $\begin{array}{l}\text { Height ratio of } \\
\text { water }\left(h_{\mathrm{w}} / h\right)\end{array}$ \\
\hline 1 & 2 & 5.33 & $1 \mathrm{e} 5$ & 0 \\
2 & 1 & & & \\
3 & 0.67 & & $1 \mathrm{e} 5$ & 0.2 \\
4 & 0.5 & 5.33 & & 0.4 \\
5 & 1 & & 0.6 \\
6 & & & 2.0 \\
7 & & & $1 \mathrm{e} 5$ & 0 \\
8 & & 0 & & \\
9 & 1 & 2.68 & & \\
10 & & 5 & & \\
11 & & 10 & $1 \mathrm{e} 4$ & \\
12 & & 5.33 & $5 \mathrm{e} 4$ & \\
13 & 1 & & $1 \mathrm{e} 5$ & \\
14 & & & & \\
15 & & & & \\
16 & & & & \\
17 & & & & \\
\hline
\end{tabular}

0.5 were chosen for analyzing the effects of the factor "height ratio of sediment $(H / h)$," while in the cases for analysis of other factor's effects, it kept as 1.0. The values of height ratio of sediment $(H / h)$ were chosen by referencing the data in the offshore area of China distributing HBS. These are shown in Table 1.

\section{Numerical results and analysis}

The distribution of shear stress at height ratio $=1$ is shown in Fig. 2a, b which is closely related with the slope failure. The data in the vertical section along slope passing through the wellbore/pipe (center section) are used for discussion. The shear stress inside the dissociation zone is larger than that outside this zone. The largest shear stress is located at the lower side of the dissociation zone (The side near the toe of slope). The reason is that the dissociation-induced softening of the dissociated zone causes its support to the surrounding soil layer decreases; meanwhile, the vertical stress and stiffness become smaller inside the dissociation zone than that outside this zone because NGH dissociation reduces the density of sediment and cementation among grains. Thus, the deformation increases and more gravity from the upper part is applied on the lower part.

The development of displacements along slope and depth at the center section when the diameter of dissociation zone is $1200 \mathrm{~m}$ is chosen for discussion (The slope is stable in this condition. The upper side of the dissociation 


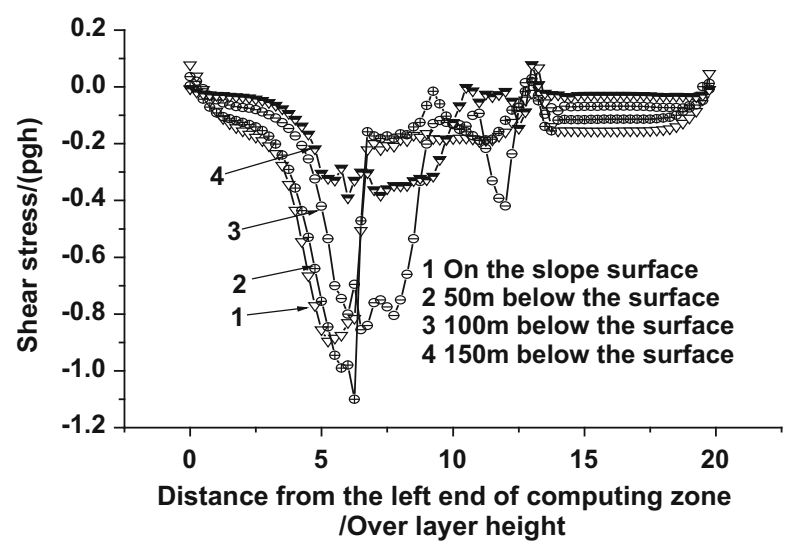

(a)

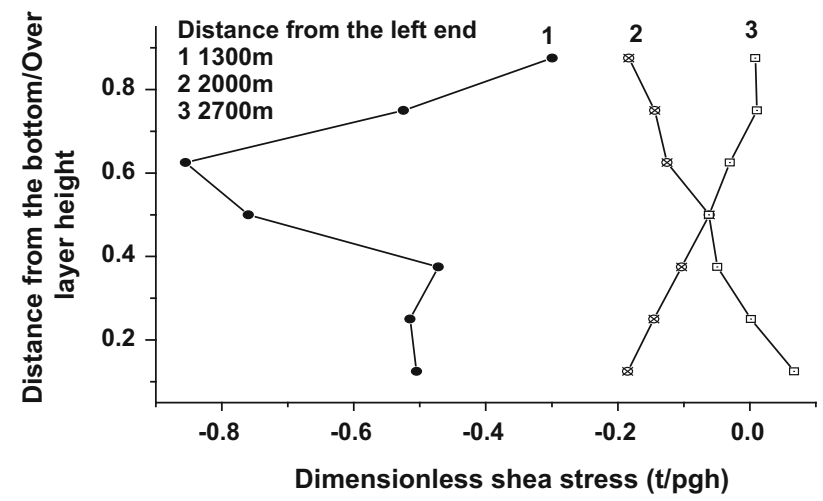

(b)

Fig. 2 Distribution of shear stress in different directions (height ratio $=1, \rho$ and $h$ are the density and height of over layer, $g$ is the gravity acceleration). a Along slope at different depths. b Along depth

zone is located at $x=2600 \mathrm{~m}$, and the lower side is located at $x=1400 \mathrm{~m}$.) (Fig 3). The vertical displacement decreases from the top to the bottom. The sediment uplifts at the lower side of the dissociation zone while it settles at the upper side (Fig. 3a). The largest horizontal displacement is located at the center of the dissociation zone $(x=2000 \mathrm{~m})$, and the displacement difference along depth is very small. The horizontal displacements near the two sides of the dissociation zone are small due to the limitation at the side interface between dissociation zone and non-dissociation zone (Fig. 3b). The sediment around the upper side of dissociation zone moves inclined downwards while it moves inclined upwards around the lower side and at the center it moves almost parallel to the slope surface. This indicates the potential sliding sediment volume and sliding interface.

From the changes of vertical displacement at the midpoint of the surface, it is shown that the displacement of the soil layer increases gradually at first with the expansion of dissociation zone. When the diameter of the dissociation zone is close to $\mathrm{CD}$, the displacement increases very fast. It

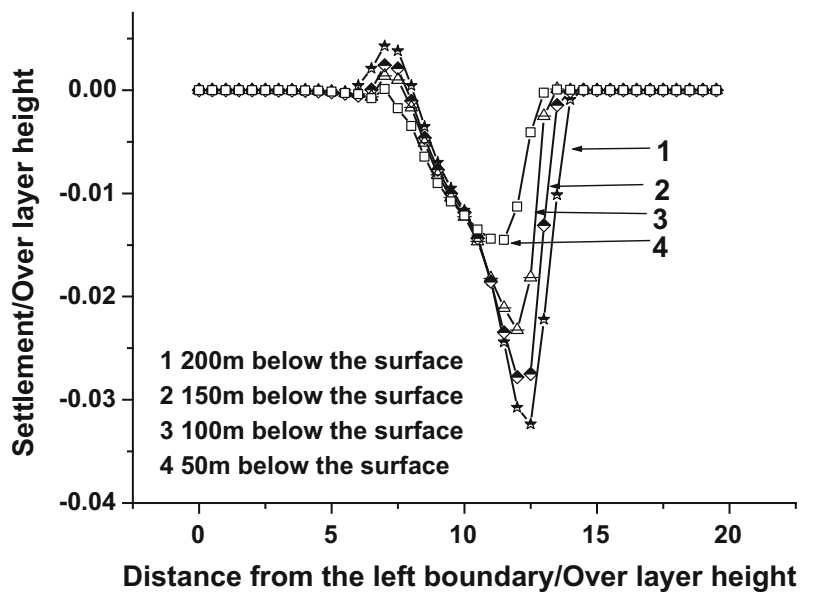

(a)

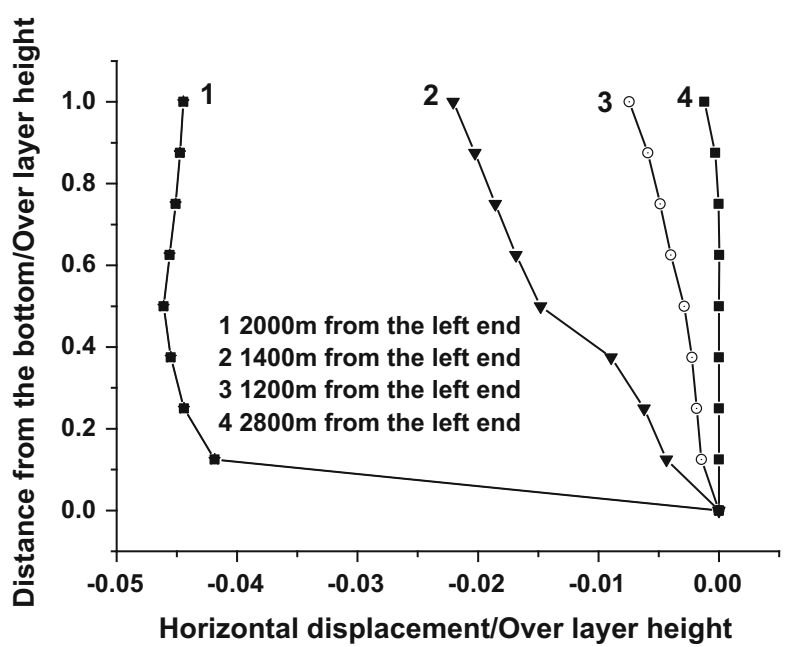

(b)

Fig. 3 Displacements in different directions. a Vertical displacement under different depths. b Horizontal displacements under vertical sections

means the development of displacement is from stable to catastrophic (Fig. 4).

\section{Effects of main dimensionless parameters on CD}

Four conditions were adopted to investigate the effects of height ratio on CD. The thickness of HBS $H$ is $100 \mathrm{~m}$, and the thicknesses of the over layer $h$ are 50,100, 150 and $200 \mathrm{~m}$, respectively. Thus, $H / h=2,1,0.67$ and 0.5 and the corresponding CDs are 900, 1400, 2200 and $2600 \mathrm{~m}$, respectively. The results show that $\mathrm{CD}$ increases with the decrease in the height ratio $H / h$ (Fig. 5 line 1). The reason is that the vertical stress applied on the HBS increases with the rise of $H / h$, and consequently, the strength of HBS and the interface both also increase; meanwhile, the over layer is just like a deep beam over HBS that the end resistance of 


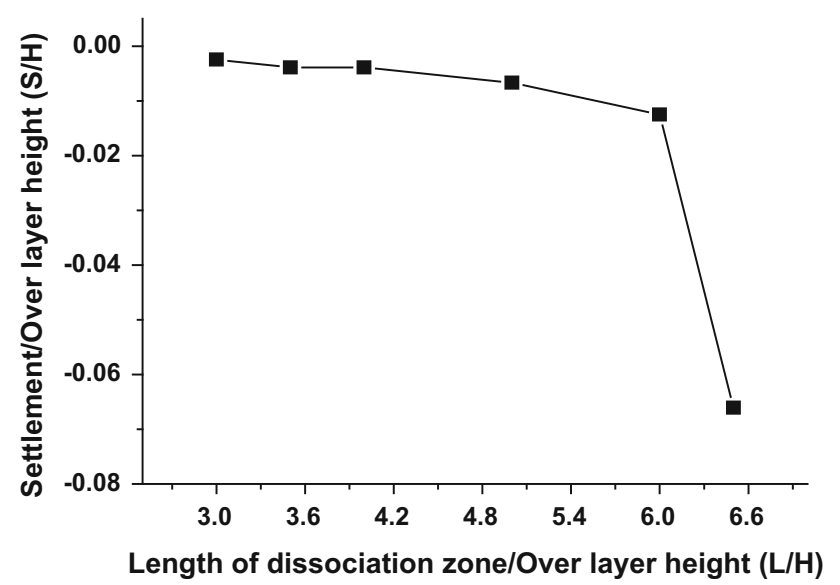

Fig. 4 Changes of vertical displacement at the center of dissociation zone with the length of dissociation zone

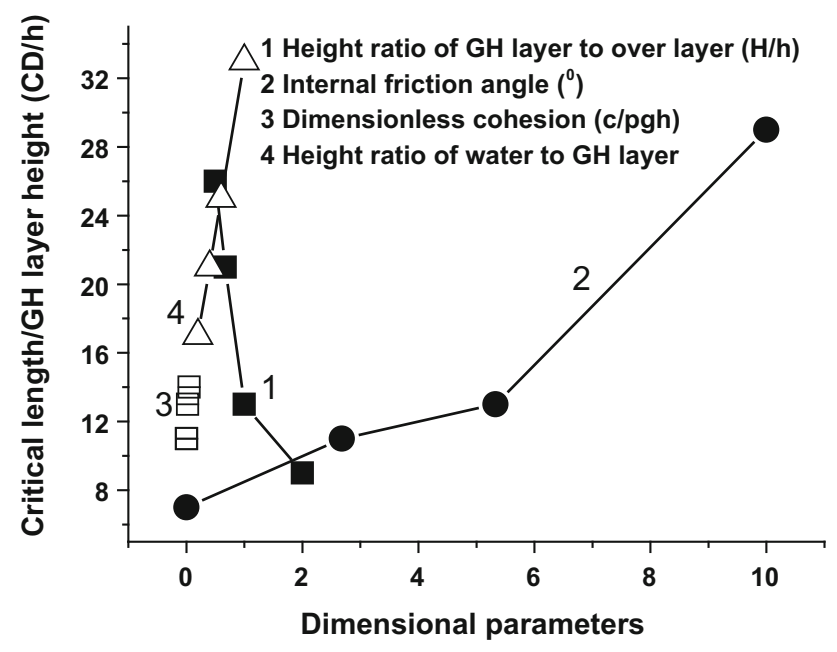

Fig. 5 Changes of critical length (CD) with dimensional parameters (height ratio of NGH layer to over layer $H / h$, internal friction angle $\varphi$ and cohesion $c /(\rho g h)$. height ratio of water to over layer $h_{\mathrm{w}} / h$,). The dots are numerical results

the potential slide surfaces must be considered; thus, CD increases.

Internal friction angle and cohesion are two parameters in Mohr-Coulomb criterion for describing the strength of sediment. According to the experimental results on the properties of HBS (Zhang et al. 2012), four values of the internal friction angle after NGH dissociation $0^{\circ}, 2.68^{\circ}$, $5.33^{\circ}$ and $10^{\circ}$ and five values of cohesion $0,1 \times 10^{4}$, $5 \times 10^{4}, 1 \times 10^{5}$ and $1 \times 10^{6} \mathrm{~Pa}$ were adopted for analyzing the effects on CD. The results are shown in Fig. 5 (lines 2, 3). It can be seen that CD becomes large with the rise of the internal friction angle and cohesion after NGH dissociation. It is because the strength of HBS increases with the rise of internal friction and cohesion according to the Mohr-Coulomb criterion. However, when the cohesion is less than some value (here it is $100 \mathrm{kPa}$ ), the decrease in cohesion has little effects on $\mathrm{CD}$. The reason is that the strength is mainly determined by the internal friction when the cohesion is less than this value.

Four conditions, $h_{\mathrm{w}} / h=0.2,0.4,0.6$ and 1.0 , were adopted to analyze the effects of water depth on CD. From Fig. 5 (line 4), it can be seen that CD increases almost linearly with water depth. Actually if the over layer is impermeable, the increase in water depth causes the rise of the normal stress at the sliding face, which induces the increase in the sediment's strength and accordingly the increase in CD. However, if the whole sediment is permeable, the increase in the water depth cannot change the effective stress; in this case, the strength will not change with the water depth that means $\mathrm{CD}$ will not be affected by the water depth if the sediment is perfect permeable.

Four elastic moduli, 5, 20, 50, 70 and $100 \mathrm{MPa}$ with $\mathrm{H} /$ $h=1$, were adopted in numerical simulation. It is shown that the elastic modulus has little effects on $\mathrm{CD}$. The reason is of the adoption of Mohr-Coulomb criterion as the failure condition which is related to the effective stress. Modulus is only related to the deformation under load. If the failure is assumed to be determined by a displacement criterion, $\mathrm{CD}$ may change with the modulus.

\section{Effects of the expansion mode of dissociation zone}

In practice, NGH dissociation can expand in different modes. For example, four modes can be imaged: (1) Mode I: If HBS is located above an oil/gas reservoir which will be recovered first, then NGH dissociation will start from the bottom of HBS due to the decrease in pressure in oil/gas reservoir and gradually expands upwards. (2) Mode II: If NGH dissociation is caused by the temperature or the level of sea water, dissociation will start from the top of HBS and expands downwards because the disturbance is transmitted from the sea water. (3) Mode III: If NGH dissociation is caused by the heat transmitted from a wellbore or vertical pipe, dissociation starts from the wellbore or pipe and expands horizontally. (4) Mode IV: If the horizontal well technique is used for NGH recovery, dissociation will starts from the middle of HBS and expands toward the top and the bottom of HBS in the mean time. The effects of these four different expansion modes (at height ratio $H / h=1.0$ ) on $\mathrm{CD}$ were investigated.

According to the numerical results, for modes I-IV, dimensionless $\mathrm{CD} / h$ are $13.5,9.5,6.51$ and 11.5 , respectively. It is shown that mode III, e.g., the dissociation mode in the whole depth of HBS and expansion in horizontal direction, is the easiest way to cause the failure of the slope, while mode I is the most difficult way to induce the sliding in the four modes discussed in this paper. It is shown in Fig. 6 that $C D$ decreases with the increase in the 


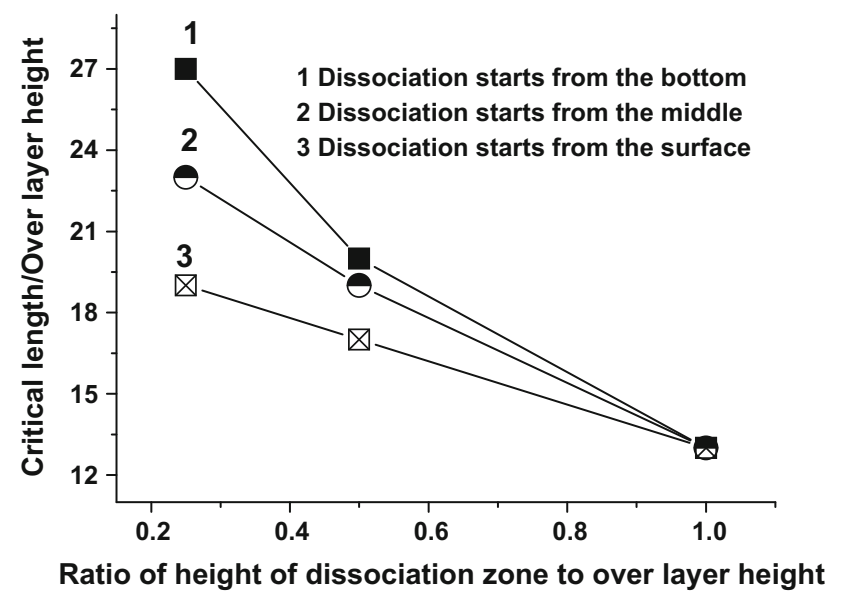

Fig. $6 \mathrm{CD}$ versus height of dissociation zone under different dissociation modes (this figure shows the relation of critical length with height of dissociation zone and dissociation ways. Three conditions are shown in this figure: $1 \mathrm{NGH}$ dissociation initiates from the bottom to the surface of HBS; $2 \mathrm{NGH}$ dissociation initiates from the middle to the surface and bottom of HBS; $3 \mathrm{NGH}$ dissociation initiates from the surface to the bottom of HBS)

dissociation zone height under different dissociation modes. The larger the height of dissociation zone is, the smaller the strength of HBS and the constraint to the over layer are. Thus, the smaller the $\mathrm{CD}$ is.

In mode I, the height of the sediment over the dissociation zone is the largest at the beginning in the four models, so the effective stress and the strength at the upper boundary of dissociation zone and the side friction of the potential slide surface are both the largest. This leads to the largest scale of dissociation zone required for providing the driving force to overcome the resistance of sliding. In model III, the HBS dissociates in the whole height, so the decrease in the modulus and strength in this zone are the largest in the four modes; consequently, the sliding resistance and CD are the smallest. In mode II, the height of the sediment over the dissociation zone is the smallest at the beginning in these four models, so the effective stress and the strength at the interface between the dissociation zone and the over layer and the side friction are both the smallest. However, the height of the dissociation zone is less than that of mode III, so the decrease in the HBS strength is smaller and the constraint to the over layer is larger than that in mode III. This leads to the larger CD in mode II than that in mode III. In mode IV, the height of the initial dissociation zone is larger than that of mode II and the other conditions are almost the same, so CD in mode IV is larger than that in mode II.

\section{Analysis based on circle arc sliding surface method}

Bishop method, one of the circle arc sliding surface methods, was used to analyze CD. In analysis, the height ratio $H / h$ was adopted as 1.0. When the safety factor was less than 1.0, the corresponding diameter of dissociation zone was thought as $\mathrm{CD}$. The safety factors at different diameters of dissociation zone are shown in Table 2. It is shown that when the diameters of the dissociation zone change from $1000 \mathrm{~m}$ to $1200 \mathrm{~m}$, the safety factors decrease from 1.046 to 0.931 , e.g., from safety to instability. From Table 2, the dissociation diameter of $1200 \mathrm{~m}$ can be taken as $\mathrm{CD}$. It has an error of $7.8 \%$ with the numerical results $(1300 \mathrm{~m})$. In other words, the numerical simulation is reliable.

\section{A simplified method for analyzing CD}

By the observation of the slope stability with NGH dissociation in experiments and the above numerical simulation, it is shown that the slide surface is often along the interface between dissociation zone and the over layer ( $\mathrm{Lu}$ et al. 2010). Because the slide is in a limited length, the friction in the over layer must be considered. Here the following assumptions are adopted: (1) The stress at the lower side of the sliding body equals the passive soil force $E_{\mathrm{p}}$. (2) The interface between dissociation zone and the over layer forms part of the slide surface. (3) It can be taken as a twodimensional problem, e.g., the critical scale of dissociation zone is much longer than the width of the over layer. (4) The effects of the pore pressure are neglected. That means, the pore fluids are drained so fast that no excess pore pressure is accumulated.

Now, the forces on the slide body include the gravity G, the resistance at the lower side $R_{\mathrm{p}}$, anti-slide force at the interface between dissociation zone and over layer $\tau_{\mathrm{f}}$, hydrostatic pressure $F$, component of the gravity, the driving force for sliding and parallel to the slope surface $T=\gamma h L \sin \theta$, component of the gravity vertical to the slope surface $N=\gamma h L \cos \theta$, in which $\gamma$ is the unit gravity, $\theta$ is the slope angle, $L$ is the length of the dissociation zone.
Table 2 Safety coefficients computed by circle arc sliding method

\begin{tabular}{lll}
\hline Dissociation length $(\mathrm{m})$ & Initial safety coefficient & Safety coefficient after NGH dissociation \\
\hline 1000 & 1.498 & 1.046 \\
1100 & 1.498 & 1.044 \\
1200 & 1.498 & 0.931 \\
\hline
\end{tabular}


Anti-slide force at the interface between dissociation zone and over layer:

$f=\tau_{f} L=\left[C_{1}+(F+N) \tan \phi_{1}\right] L$

in which $\mathrm{C}_{1}$ is the cohesion at the interface, $F=\rho_{\mathrm{w}} g h_{\mathrm{w}}=\gamma_{\mathrm{w}} h_{\mathrm{w}}$.

The passive force at the lower side of the over layer $R_{\mathrm{p}}$.

$\mathrm{R}_{p}=0.5 \gamma h^{2} k_{\mathrm{p}}+2 c h \sqrt{k_{\mathrm{p}}}$

in which $k_{\mathrm{p}}=\tan ^{2}\left(45^{\circ}+0.5 \varphi\right), c$ is the cohesion and $\varphi$ is the internal friction angle at the interface between dissociation zone and over layer, $h$ is the height of the over layer.

When the size of dissociation zone arrives at CD:

$\frac{F+R_{\mathrm{p}}}{T}=1$

So the $\mathrm{CD}(L)$ can be solved as.

$L=\frac{R_{\mathrm{p}}}{T-f}=\frac{0.5 \gamma h^{2} k_{\mathrm{p}}+2 c h \sqrt{k_{\mathrm{p}}}}{\gamma h \sin \theta-C_{1}-\left(\gamma_{\mathrm{w}} h_{\mathrm{w}}+\gamma h \cos \theta\right) \tan \phi_{1}}$

To certificate the theoretical $\mathrm{CD}$, compare it with the above numerical results. The results show that the two results are close to each other with a variance of less than $10 \%$ which comes from the simplification and the four assumptions in the theoretical analysis and the inevitable error from numerical simulation (Fig. 7).

\section{Comparison of centrifugal test results and numerical results}

The centrifugal test was carried out in a box with a size of $60 \mathrm{~cm}$ in length, $35 \mathrm{~cm}$ in width and $40 \mathrm{~cm}$ in height. Silty soil was used as the material of over layer (located above NGH layer) with specific gravity 2.75 , dry density $1300 \mathrm{~kg} / \mathrm{m}^{3}$, porosity $52 \%$ and water content $34 \%$. The slope angle was $14^{\circ}$. Clay with dry density of $1100 \mathrm{~kg} / \mathrm{m}^{3}$, and porosity of $60 \%$ was used as the skeleton of HBS. The thicknesses of HBS and the over layer were 15 and $7 \mathrm{~cm}$, respectively. Triaxial tests were conducted under undrained and unconsolidated conditions (UU) to measure the mechanical parameters. The internal friction angle and cohesion of the over layer are $0.6^{\circ}$ and $35 \mathrm{kPa}$ under confining pressures of $50-200 \mathrm{kPa}$. The internal friction angle and cohesion of HBS change from $0.8^{\circ}$ to $80 \mathrm{kPa}$ before NGH dissociation to $0.5^{\circ}$ and $7 \mathrm{kPa}$ after totally dissociation, respectively. The detailed parameters of the material are listed in Table 3.

During tests, the maximum acceleration was $150 \mathrm{~g}$. The pore pressure, deformation and temperature were recorded. In this section, deformation of the surface was used for

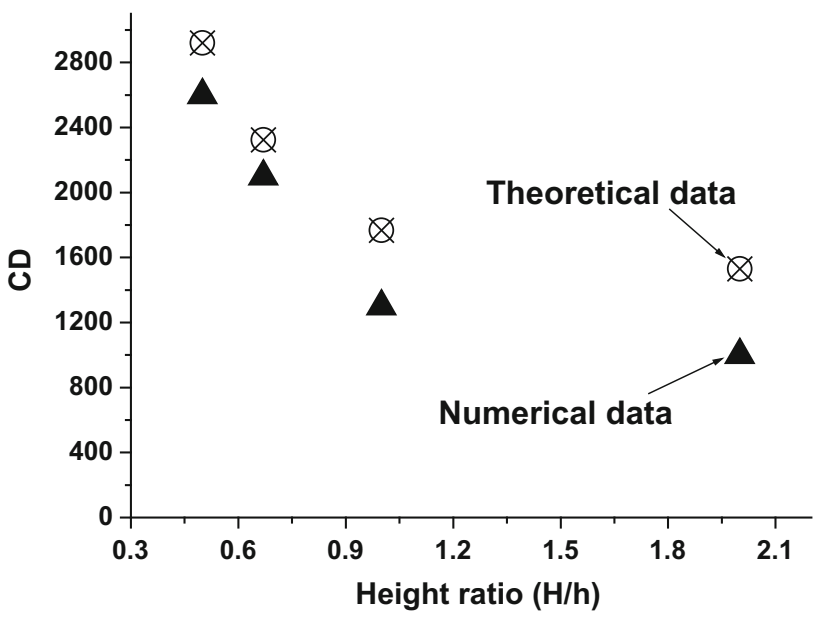

(a)

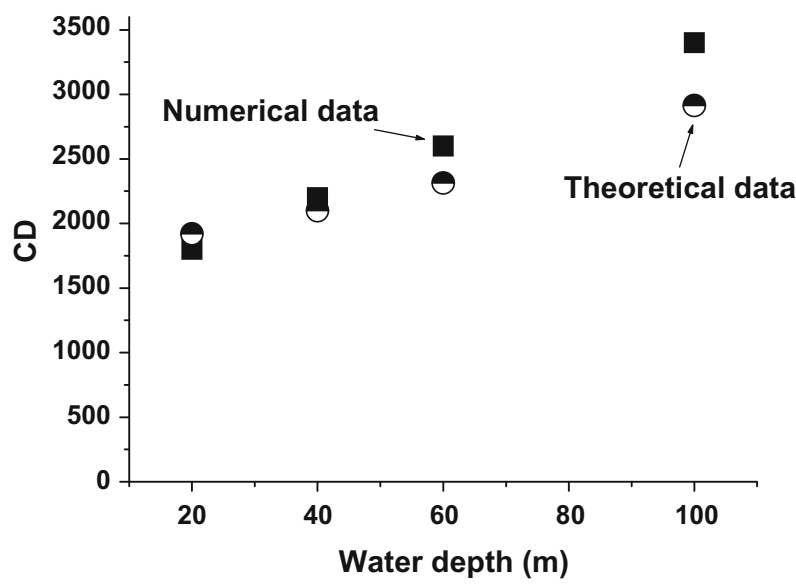

(b)

Fig. 7 Comparison of theoretical and numerical results of $C D$ versus height ratio $(H / h)$. a Critical length under different height ratio of NGH layer to over layer. b Critical length under different water depth

Table 3 Parameters of materials used in experiments

\begin{tabular}{lll}
\hline & $\begin{array}{c}\text { Over } \\
\text { layer }\end{array}$ & NGH layer \\
\hline Dry density $\left(\mathrm{kg} / \mathrm{m}^{3}\right)$ & 1300 & 1100 \\
Porosity $(\%)$ & 52 & $60 \%$ \\
Water content $(\%)$ & 34 & Saturated with \\
& & TFH solution \\
Slope angle $\left(^{\circ}\right)$ & 14 & 14 \\
Thicknesses $(\mathrm{cm})$ & 7 & 15 \\
Internal friction angle $\left({ }^{\circ}\right)($ confining & 0.6 & Before \\
pressures of 50-200 kPa) & & dissociation 0.8 \\
& & After dissociation \\
& & 0.5 \\
Cohesion $(\mathrm{kPa})($ confining pressures of & 35 & Before \\
$50-200 \mathrm{kPa})$ & & dissociation 80 \\
& & After dissociation \\
& & 7 \\
\hline
\end{tabular}




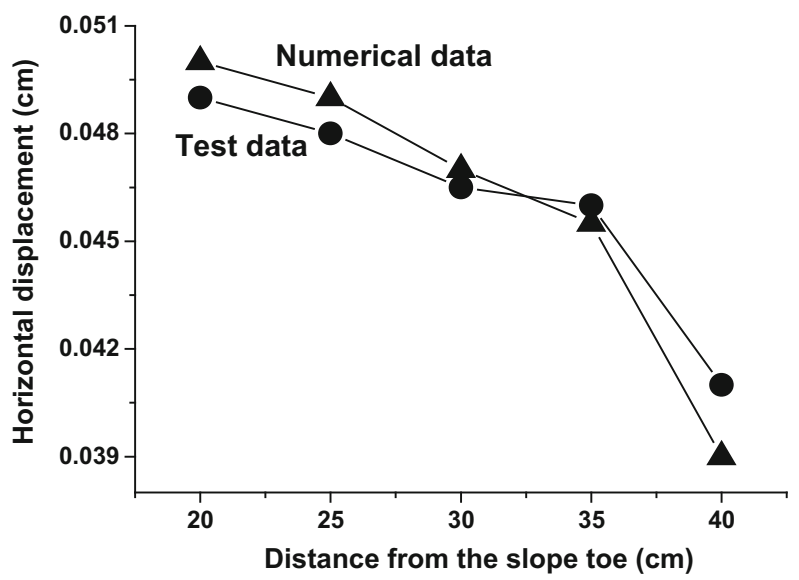

(a)

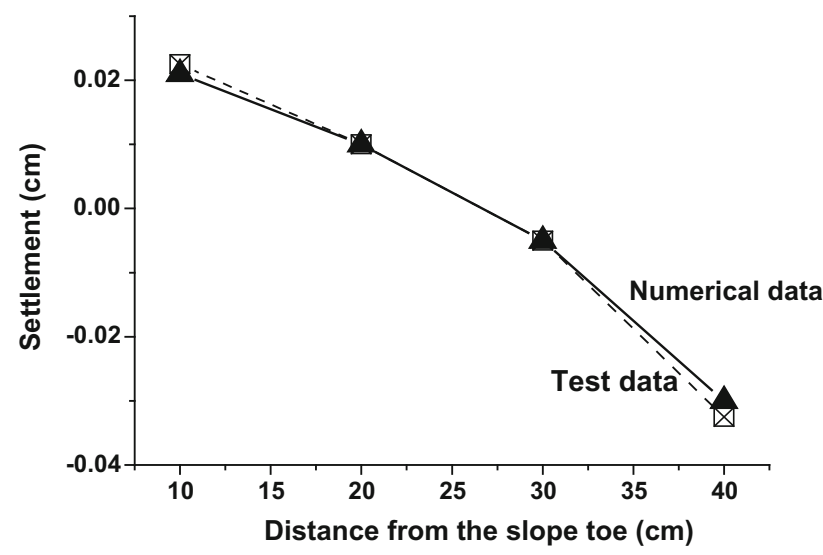

(b)

Fig. 8 Comparison between numerical and centrifugal results of the deformation on the slope surface. a Comparison of horizontal displacement. b Comparison of settlement

comparison. From Fig. 8, it can be seen that the numerical results and the centrifugal data in the middle part are close to each other. The difference at the two ends is caused by the boundary effects (Zhang et al. 2015).

\section{Conclusions}

Deformation and sliding of the sediment after dissociation of NGH have been numerically investigated. Main conclusions are as follows:

1. There is a critical scale of dissociation zone (CD) over which there will be landslide. $\mathrm{CD}$ is smaller when NGH dissociation zone expands along the slope with the whole height of HBS than when it expands upwards with a horizontal section.

2. A simplified method for solving $\mathrm{CD}$ was presented and compared with the numerical results.
3. The main factors affecting the stability of marine slope after NGH dissociation are height ratio of the NGH layer to the over layer, ratio of the sea water depth to the thickness of NGH layer, strength of sediment after NGH dissociation.

Acknowledgements This paper is supported by The National Natural Science Foundation of China (Nos. 51239010, 11272314) and Project of CAS-CNPC Strategic Alliance.

\section{References}

Briaud JL, Chaouch A (1997) Hydrate melting in hydrate soil around hot conductor. J Geotech Geoenviron Eng 123(7):645-653

Brooks JM, Cox BH, Bryant WR, Kennicutt MC II, Mann RG, McDonald TJ (1986) Association of gas hydrates and oil seepage in the Gulf of Mexico. Org Geochem 10(1-3):221-234

Bugge T, Befring S, Belderson RH, Eidvin T, Jansen E, Kenyon NH, Holtedahl H, Sejrup HP (1987) A giant three-stage submarine slide off Norway. Geo-Mar Lett 7(4):191-198

Chaouch A, Briaud JL (1997) Post melting behavior of gas hydrates in soft ocean Sediments. In: offshore technology conference (OTC8298), pp 1-11

Jung WY, Peter RV (2004) Effects of bottom water warming and sea level rise on Holocene hydrate dissociation and mass wasting along the Norwegian-Barents continental Margin. J Geophys Res 109(B6):B06104

Kwon TH, Cho GC, Santamarina JC (2008) Gas hydrate dissociation in sediments: pressure-temperature evolution. Geochem Geophys Geosystem 9(2):Q03019

Li XP, He SM (2012) Progress in stability analysis of submarine slopes considering dissociation of gas hydrate. Environ Earth Sci 66(3):741-747

Liu LL, Lu XB, Zhang XH (2015) A theoretical model for predicting the spatial distribution of gas hydrate dissociation under the combination of depressurization and heating without the discontinuous interface assumption. J Petrol Sci Eng 133:589-601

Lu XB, Wang L, Wang SY, Li QP (2008) Study on the mechanical properties of THF hydrate Deposit. In: Proceedings of 18th international offshore and polar engineering conference, vancouver, canada, ISOPE1, pp 57-60

Lu XB, Li QP, Wang L (2010) Instability of seabed and pipes induced by NGH dissociation, In: Proceedings of 20th International Offshore and Polar Engineering Conference, Beijing, China, ISOPE, vol, 1pp 110-114

Marcia KM, Rich C, Timothy JC, George DG, Paul AH, Thomas BR, Omer S, Frank S (2012) Review of flow rate estimates of the deepwater horizon oil spill. Proc Natl Acad Sci 109(50):20260-20267

Milkov AV (2000) Worldwide distribution of submarine mud volcanoes and associated gas hydrates. Mar Geol 167:29-42

Sloan ED (1998) Clathrates of natural gases. Dekker, New York, p 705

Wang SY, Zheng W, Lu XB (2009) The effects of gas hydrate dissociation on the stability of pipeline in seabed. In: Proceedings of 19th international offshore and polar engineering conference, Osaka, Japan, 1: 49-53

Xu W, Germanovich LN (2006) Excess pore pressure resulting from methane hydrate dissociation in marine sediments: a theoretical approach. J Geophys Res 111:B011104 
Zhang XH, Lu XB, Li QP (2010) Thermally induced evolution of phase transformations in gas hydrate sediment. Sci China Phys Mech Astron 53(8):1530-1535

Zhang XH, Lu XB, Zheng ZM (2011) Layered fracture and outburst due to dissociation of hydrate. J Petrol Sci Eng 76:212-216

Zhang XH, Lu XB, Zhang LM, Wang SY, Li QP (2012) Experimental study on mechanical properties of methane-hydrate-bearing sediments. Acta Mech Sin 28(5):1-11
Zhang XH, Lu XB, Shi YH, Xia Z, Liu WT (2015) Centrifuge experimental study on instability of seabed stratum caused by gas hydrate dissociation. Ocean Eng 105:1-9

Zheng ZC, Chen JR, Zhu ZY (2004) Physical and mechanical characteristics of seabed soils and its geological environment in South China Sea. Hydrogeol Eng Geol 31(4):50-53 (in Chinese) 